\title{
Focusing on Preparing Newly-qualified Doctors for Their On-calls Using Peer-assisted Learning
}

\author{
Faheem Malik $^{*}$
}

\author{
Oxford University Hospitals NHS Foundation Trust \\ *faheemmalik@hotmail.co.uk
}

\begin{abstract}
Teaching for newly-qualified Foundation Year 1 (FY1) doctors at our hospital has traditionally been delivered by senior clinicians. They have tended to focus on specialised and advanced areas of their fields, rather than the more basic pathologies encountered by a junior doctor on-call. This was resulting in reduced confidence amongst FY1 doctors. To solve this problem, we created a teaching course using a peer-assisted learning (PAL) approach. It was delivered by fellow trainees who better understood the learning needs of FY1 doctors. We compared the cohort of newly-qualified doctors that received the course (2020/21) with the preceding cohort who did not receive the course (2019/20). The $2020 / 21$ cohort reported a significant increase in confidence in dealing with different specialties on-call compared to the 2019/20 cohort ( $<<0.0001$ ). As a result of our project, our hospital has incorporated this course into core-teaching for FY1 doctors at the beginning of every year.
\end{abstract}

Keywords: "Peer-assisted learning".

\section{INTRODUCTION}

We noticed two problems with the teaching being given to us and our fellow newly-qualified Foundation Year 1 (FY1) doctors at our hospital. Firstly, that it did not focus on common conditions encountered by junior doctors on-call. Secondly, that it did not focus on the practical aspects of being on-call.

Feedback on existing teaching had highlighted one possible reason for this: our teachers were usually senior clinicians, who tended to focus on pathologies or scenarios more commonly encountered by them rather than by an FY1. For example, a Consultant Cardiologist may choose to teach about the intricacies of cardiac catheterisation. However, the management of acute heart failure, which may seem basic and bland to a specialist, is more relevant and useful to the newly-qualified doctor. Furthermore, practical knowledge that is useful whilst on-call may not be readily available to senior clinicians. Many tasks that are important to an FY1 on-call, such as updating patient lists, are rarely carried out by Consultants. Thus, senior clinicians are not always best placed to deliver teaching on the practical aspects of being on-call.

The result was that FY1 doctors at our hospital felt they lacked confidence in their medical and practical knowledge whilst on-call. We decided to solve these problems by designing a teaching programme using a peer-assisted learning (PAL) approach.

PAL refers to "people from similar social groupings who are not professional teachers helping each other to learn and learning themselves by teaching'.[1] It requires teachers and learners to be of a similar level, and is utilised in undergraduate[2] and postgraduate[3] medical education. We hypothesised that this approach would be more effective than existing FY1 teaching for one main reason: it is easier to tailor teaching to the needs of the learner if it is delivered and organised by teachers of a similar level and experience.

We set out to create a teaching programme that would be delivered by near-peers (fellow trainee doctors) rather than Consultants, with a specific focus on preparing FY1s for their on-calls. This article describes and evaluates our programme.

\section{METHODS}

We decided to compare two different cohorts of newly-qualified doctors. The first cohort (our own) carried out their FY1 year in 2019/20 and did not receive our teaching programme. The second cohort carried out their FY1 year in 2020/21 and did receive our teaching programme. 
We distributed a pre-programme questionnaire to the 2019/20 cohort in December 2019. Its first purpose was to assess and quantify our assumption that FY1 doctors lacked confidence in dealing with common conditions on-call. This allowed us to demonstrate the need, or not, for our programme. Its second purpose was to assess the 2019/20 cohort's confidence, having not received our teaching programme, in dealing with on-call presentations and emergencies in 4 specialties that newly-qualified doctors are commonly exposed to oncall: General Surgery, Nephrology, Gastroenterology, and Cardiology. We asked for a rating of 0 (no confidence) to 5 (complete confidence).

Over the next 8 months we created our teaching programme. We proposed the following imperatives: (1) To conform with a PAL-based approach teaching sessions must be delivered by near-peers (Foundation Year doctors, Core Trainees, or Specialty Registrars) rather than Consultants, and (2) Teaching must focus on conditions and scenarios an FY1 would feasibly encounter on-call. Additionally, to facilitate increasing FY1 doctors' confidence in dealing with the practicalities of on-calls, we created a dedicated teaching session at the beginning of the course. It covered a number of topics, including where to find different medications on the wards, how to update lists, and who to escalate to when in need of help. The complete programme consisted of 5 sessions:

1. A guide to the practicalities of on-calls at our hospital

2. A guide to General Surgery on-call

3. A guide to Nephrology on-call

4. A guide to Gastroenterology on-call

5. A guide to Cardiology on-call

We delivered this programme to the next cohort (2020/21) of newly-qualified doctors at the beginning of their FY1 year.

A post-programme questionnaire was sent out to the 2020/21 cohort in December 2020 after they had completed our teaching programme. We distributed this at the same time of year as the pre-programme questionnaire was distributed to the 2019/20 cohort to allow direct comparison between the two groups at the same point in their training. As in the pre-programme questionnaire, we assessed confidence in dealing with on-call presentations and emergencies in General Surgery, Nephrology, Gastroenterology, and Cardiology. We used the same rating system of 0 (no confidence) to 5 (complete confidence). We also made an additional assessment of global confidence with on-calls before and after the programme, allowing us to calculate an intracohort change in rating.
We compared the results of the pre-programme questionnaire with the results of the post-programme questionnaire. This allowed us to assess differences in confidence between FY1 doctors who had received our teaching programme and those who had not.

Confidence ratings were roughly normally distributed, allowing us to carry out statistical analysis using the unpaired $t$ test. We calculated the significance of any difference between the 2019/20 cohort and the 2020/21 cohort in each individual specialty using the mean confidence ratings and standard deviations of each cohort for each specialty. We also calculated the significance of any difference between the cohorts as a whole, collating results for all four specialties, using the assumption that each individual's ratings in the four different specialties were independent from one another. We believe this is a valid assumption given that the four specialties in our programme are unrelated, and so should not result in clustering of an individual's confidence ratings.

\section{Results}

\subsection{Pre-programme questionnaire}

39 FY1 doctors from the 2019/20 cohort responded to the pre-programme questionnaire.

Our first question sought to determine whether or not a teaching programme was necessary at all. We asked "Is there a need for more teaching on acute presentations encountered whilst on-call?". FY1 doctors responded with a rating of $0-5$, with $0 / 5$ being equivalent to "completely disagree" and $5 / 5$ being "completely agree". The cohort gave an average rating of $4.51 / 5$, with $64.1 \%$ of doctors choosing a maximum rating of 5. These results gave us enough evidence to justify delivering our teaching programme.

Our second question asked the following: "How confident do you feel about common on-call presentations and emergencies in the following areas? (1) General Surgery, (2) Nephrology, (3) Gastroenterology, and (4) Cardiology". Average confidence ratings for each specialty were calculated (Figure 1). The overall average confidence rating for FY1 doctors who had not received our teaching programme was 2.09/5.

\subsection{Post-programme questionnaire}

18 FY1 doctors from the 2020/21 cohort responded to the post-programme questionnaire.

As in the pre-programme questionnaire, we asked the following question: "How confident do you feel about common on-call presentations and emergencies in the following areas? (1) General Surgery, (2) Nephrology, (3) Gastroenterology, and (4) Cardiology". Average 


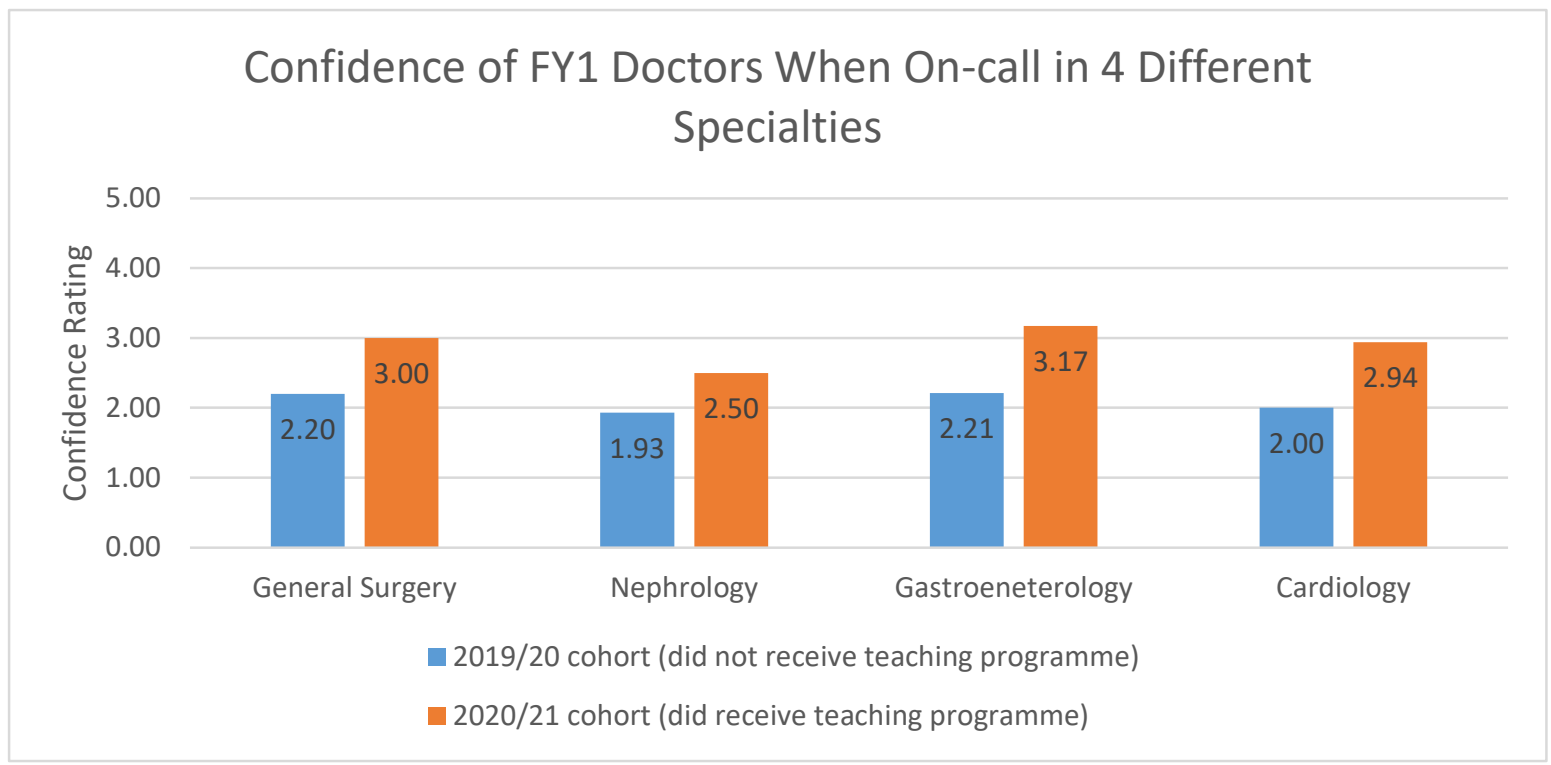

Figure SEQ Figure 1* ARABIC 1: Comparing the confidence ratings of the 2019/20 cohort in 4 different specialties with the confidence ratings of the 2020/21 cohort

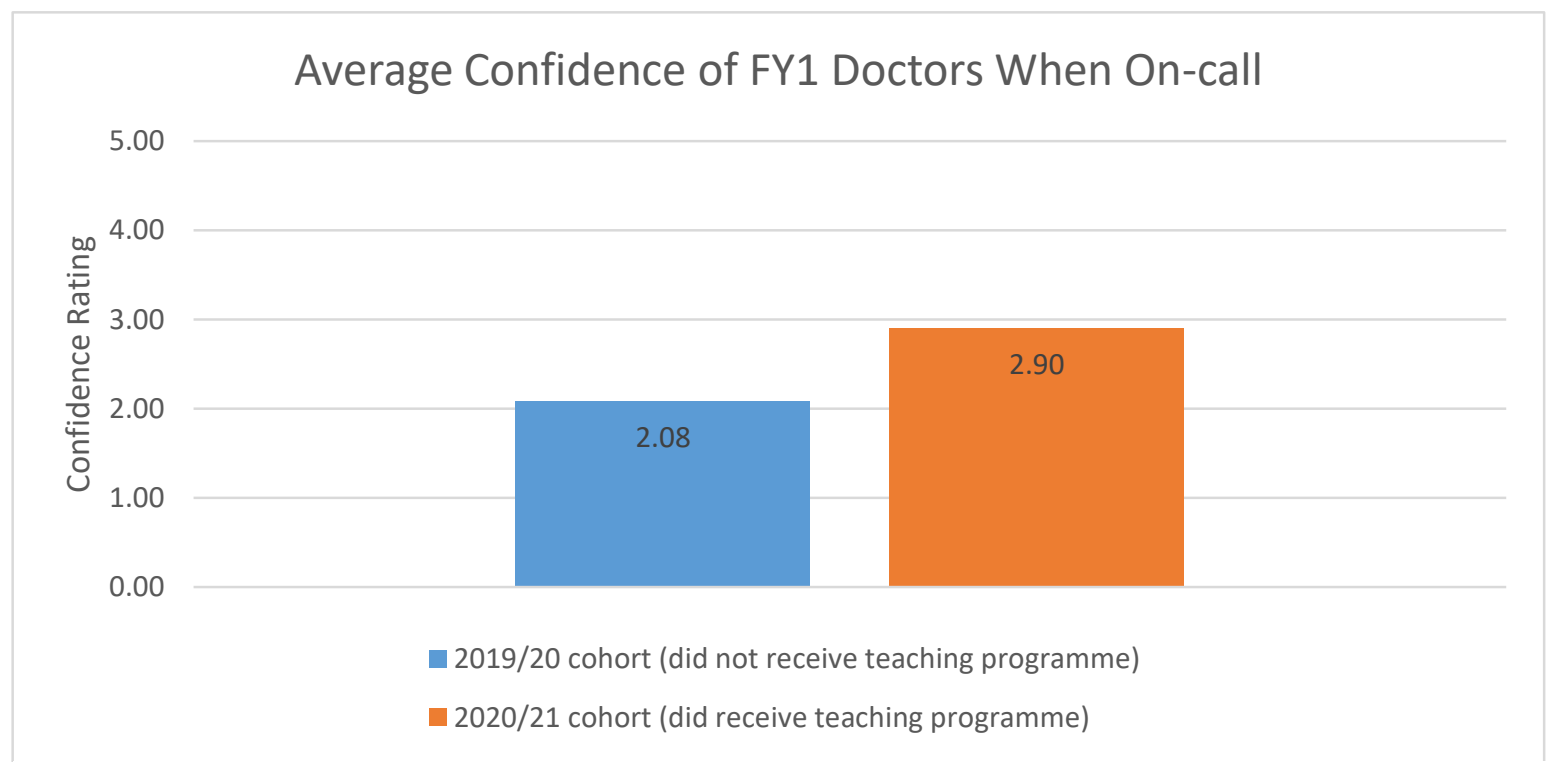

Figure 2: Comparing the confidence ratings of the 2019/20 cohort with the 2020/21 cohort when rating for each specialty were combined and averaged

confidence ratings for each specialty were calculated (Figure 1). The overall average confidence rating for FY1 doctors who had received our teaching programme was $2.90 / 5$.

We also asked this cohort to give a global confidence rating for dealing with common conditions on-call before and after the teaching programme. On average, they reported a confidence of $1.78 / 5$ before receiving our teaching and 3.56/5 after receiving our teaching. This demonstrates a marked intra-cohort increase in confidence as a result of our programme.

\subsection{Overall results}

We compared the 2020/21 cohort, who did receive our teaching programme, to the 2019/20 cohort, who did not receive our teaching programme. There was a statistically significant increase in confidence in the $2020 / 21$ cohort in General Surgery $(\mathrm{p}=0.03)$, Gastroenterology $(\mathrm{p}=0.003)$ and Cardiology $(\mathrm{p}=0.007)$. The increase in confidence in Nephrology did not quite meet significance $(\mathrm{p}=0.08)$.

When confidence ratings for all four specialties were collated, the 2020/21 cohort gave an overall average rating of 2.90/5 while the 2019/20 cohort gave an overall 
average rating of 2.08/5 (Figure 2). The increase in confidence in the cohort that received our teaching course was statistically significant $(\mathrm{P}<0.0001)$.

We asked for these ratings at the same point in both cohort's training to control for level of clinical experience.

\section{DISCUSSION}

Our results demonstrate that providing newlyqualified FY1 doctors with teaching delivered by nearpeers, and focused specifically on preparing them for their on-calls, significantly improves their confidence in dealing with common on-call presentations and emergencies.

We attribute our programme's success to the incorporation of PAL techniques. We believe that nearpeers were much better able to understand the educational needs of our learners because their knowledge and experience did not differ too significantly from FY1 doctors'. This phenomenon, termed 'cognitive congruence' in the PAL literature,[4-6] allowed our teachers to tailor their sessions appropriately and focus on what was important to a newly-qualified doctor. We also discovered, through feedback received after the programme, that FY1 doctors felt more comfortable giving constructive feedback to near-peers than to senior clinicians - a finding that has been repeatedly demonstrated elsewhere.[7] This further facilitates understanding of learning needs.

It has been proposed that PAL reduces the workload for faculty members and senior staff.[8,9] While we did not formally quantify this in our own programme, it is clear that there was a significant reduction in the need for senior clinician involvement in FY1 teaching throughout the duration of our teaching course. Sessions were organised by ourselves and delivered by near-peers, with Consultant input required only for approval of the programme.

Using near-peers to deliver teaching, rather than senior clinicians, has the additional benefit of providing a learning opportunity for the teacher as well as the learner.[10] Trainees who took part in our teaching programme had their own knowledge tested, and were given the chance to develop their teaching skills in a constructive and friendly environment. Developing these skills has been highlighted as a core component of the Foundation Programme Curriculum for newly-qualified doctors in the UK.[11]

Going forwards, we would like to assess two further outcomes. Firstly, whether or not the increase in confidence our teaching programme gives FY1 doctors translates into an improvement in patient care; secondly, the degree to which senior clinician workload is reduced by our teaching programme.

\section{CONCLUSION}

We have demonstrated that using a PAL approach focused on preparing newly-qualified FY1 doctors for their on-calls significantly increases their confidence. We have identified the following benefits of PAL:

1. Teaching is more relevant to learners because teachers have a better understanding of their own (and thus their peers') learning needs.

2. It lightens the teaching burden on busy senior clinicians, and thus increases the chance that hospitals will adopt PAL-based teaching programmes long-term.

3. Learning is bidirectional, with both teachers and learners benefitting from the experience.

\section{AUTHORS' CONTRIBUTIONS}

F.M. and T.T. conceived, organised, and helped deliver the teaching programme.

F.M. collected, analysed and interpreted the data.

F.M. wrote the abstract and manuscript.

F.M. and T.T. approved the final version of the manuscript to be published.

\section{REFERENCES}

[1] Topping KJ. The effectiveness of peer tutoring in further and higher education: A typology and review of the literature. High Educ 1996;32:32145. doi:10.1007/BF00138870

[2] Ross MT, Cameron HS. Peer assisted learning: a planning and implementation framework: AMEE Guide no. 30. Med Teach 2007;29:527-45. doi:10.1080/01421590701665886

[3] Thampy H, Kersey N. Peer-assisted learning for foundation doctors. Clin Teach 2017;14:180-3. doi:10.1111/tct.12586

[4] Loda T, Erschens R, Loenneker H, et al. Cognitive and social congruence in peer-assisted learning - A scoping review. PLoS One 2019;14. doi:10.1371/journal.pone.0222224

[5] Loda T, Erschens R, Nikendei C, et al. A novel instrument of cognitive and social congruence 
within peer-assisted learning in medical training: construction of a questionnaire by factor analyses. BMC Medical Education 2020;20:214. doi:10.1186/s12909-020-02129-x

[6] Lockspeiser TM, O’Sullivan P, Teherani A, et al. Understanding the experience of being taught by peers: the value of social and cognitive congruence. Adv Health Sci Educ Theory Pract 2008;13:361-72. doi:10.1007/s10459-006-9049-8

[7] Topping KJ. Trends in Peer Learning. Educational Psychology 2005;25:631-45. doi:10.1080/01443410500345172

[8] Peets AD, Coderre S, Wright B, et al. Involvement in teaching improves learning in medical students: a randomized cross-over study. BMC Med Educ 2009;9:55. doi:10.1186/1472-6920-9-55

[9] Roberts NJ, Brockington S, Doyle E, et al. Pilot study of an innovative model for clinical education in dietetics. Nutrition \& Dietetics 2009;66:39-46. doi:https://doi.org/10.1111/j.17470080.2008.01316.x

[10] Weiss V, Needlman R. To teach is to learn twice. Resident teachers learn more. Arch Pediatr Adolesc Med 1998;152:190-2. doi:10.1001/archpedi.152.2.190

[11] The Foundation Programme Curriculum 2016. https://foundationprogramme.nhs.uk/curriculum/ 